\title{
Scleractinian Corals and Reefs of Vietnam as a Part of the Pacific Reef Ecosystem
}

\author{
Yuri Ya Latypov \\ A. V. Zhirmunsky Institute of Marine Biology, Far East Branch of the Russian Academy of Sciences, Vladivostok, Russia \\ E-mail:ltpv@mail.ru \\ Received May 12, 2011; revised June 1, 2011; accepted June 10, 2011
}

\begin{abstract}
The paper analyzes both published and unpublished results of the investigations of Vietnamese reef building corals and reefs performed in the last decades of the twentieth century and first decades twenty-first. The state of the art in the study of reef-building scleractinian corals and reefs is presented. The scleractinian fauna of Vietnam is shown to match in species diversity (350 species of 80 genera) the tropical coral fauna of the Indonesian-Malacca fertile center, from which Indo-Pacific reef-building corals originated. The whole Vietnam coast from the Gulf of Tonkin to the Gulf of Siam is a biogeographically single whole and is a part of the Indo-Polynesian Province of the Indo-Pacific Area.
\end{abstract}

Keywords: Vietnam, Reefs, Reef-Building Corals, Pacific Reef Ecosystem

\section{Introduction}

\subsection{Brief the Ecological Characteristic and a History of Studying}

The coastline of Vietnam is over $3200 \mathrm{~km}$ long and covers 15 degrees in latitude, from the Gulf of Siam in the south $\left(8^{\circ} \mathrm{N}\right)$ to the Chinese border in the north $\left(23^{\circ} \mathrm{N}\right)$. The near shore water area (up to 50-m deep) of Vietnam, including some 3000 islands, is about 206,000 km². Vietnam and its coastline are divided into 5 parts, the Gulf of Tonkin, Central and Southern Vietnam, Gulf of Siam, and Spratly Islands [1]. Reef-building corals and reef accumulations are confined to hard grounds, typical of the Vietnam coast. Between $16^{\circ}$ and $19^{\circ} \mathrm{N}$, the coastline is formed mostly by moving sand with a minor presence of hard substrates. The temperature varies between $18^{\circ} \mathrm{C}-32^{\circ} \mathrm{C}$, and the salinity, $28 \%$ - $40 \%$. One hundred and fourteen rivers are registered along the coastline. The spread of the reef is limited near the mouths of two large rivers, the Red River in the north and the Mekong in the south, due to adverse conditions. The ecosystems of the coral reefs of Vietnam feature high bioproductivity, with a primary production of up to $30-100 \mathrm{mg} \cdot \mathrm{C} / \mathrm{m}^{3}$ per day, which is almost 100 times that in open waters $[2,3]$.

Vietnam is situated in the tropics, affected by two sorts of monsoons: the wet southwest, lasting from May till September, and the dry northeast, occurring in October-April. Heavy rain showers during the wet monsoon period result in a huge $\left(5-400\right.$ million $\left.\mathrm{m}^{3}\right)$ freshwater influx and a substantial (up to 200 thous. tons) terrigenous sediment influx into the sea. The daily suspended matter precipitation rate in the reef reaches $70-100 \mathrm{~g} / \mathrm{m}^{2}$ and increases tenfold during typhoons [4,5]. This results in a remarkable decrease in water transparency, affecting, together with other factors, the development of coral settlement in this region.

The reef-building corals and reefs of Vietnam attracted scientific attention as early as the first half of the twentieth century [6-8] was the first to analyze the zonation of reef-building corals in reefs of the Khanh Hoa province. He distinguished four scleractinian-dominated facieses. These investigators determined the species composition of scleractinians and demonstrated its similarity to that of Australia and Indonesia. Beginning in 1980, systematic studies of Vietnam corals and reefs have been performed in joint expeditions by the Institute of Marine Biology (Vladivostok), Nha Trang Institute of Oceanography, Haiphong Institute of Oceanology, and WWF (World Wide Fund for Nature). The published results were mainly related to scleractinian composition and distribution, with some papers analyzing common accompanying macrobenthos species and a few publications providing the general characteristics of the reefs. Part of the data obtained was presented only in unpublished reports. 
Some findings were published in difficult-to obtain regional works, including Vietnamese ones.

To date, in a region bordered by the Gulf of Tonkin in the north, the Gulf of Siam in the south, and the Spratly Islands in the South China Sea, all reef-building areas including large islands and shoals have been studied (Figure 1).

It is thus topical to review the data available on the coral composition and distribution and development of Vietnam's reefs, as well as on their relation to the IndoPacific reef ecosystem. The rapid growth of the country's population of 70 million, together with travel industry development and marine aquaculture intensification [9], has resulted in increased anthropogenic pressure on this unique ecosystem. Generalizing data on the composition, structure, and environmental conditions of Vietnam's reef communities will allow one to evaluate the degree of their degradation and the outlook for their conservation and recovery.

\subsection{Morphological Features of the Vietnamese Reefs}

Both along the coastline and around the islands, reefbuilding corals form diverse reef topographies. These include small fringing reefs along the coastline, barrier reefs separated from the continent (Re Island and Jang Bo Reef), and atolls (Spratly Islands) in the open South China Sea [10-14]. Vo and Hodgson [5] reported Vietnam's reefs to include both true reef frameworks and coral gardens. Various calcareous structures occurring on reefs might be formed by coral settlements, usually called coral layers, communities, or specialized settle- ments. Such structures are typical of early reef development stages and lack any geomorphological and verticalbiological zonation [15-19]. All Vietnam reefs display distinct biological and more or less prominent morphostructural zonation. By the specificity of geomorphological and climate conditions, Vietnam's fringing reefs are clearly distinguished into two types (Figure 2).

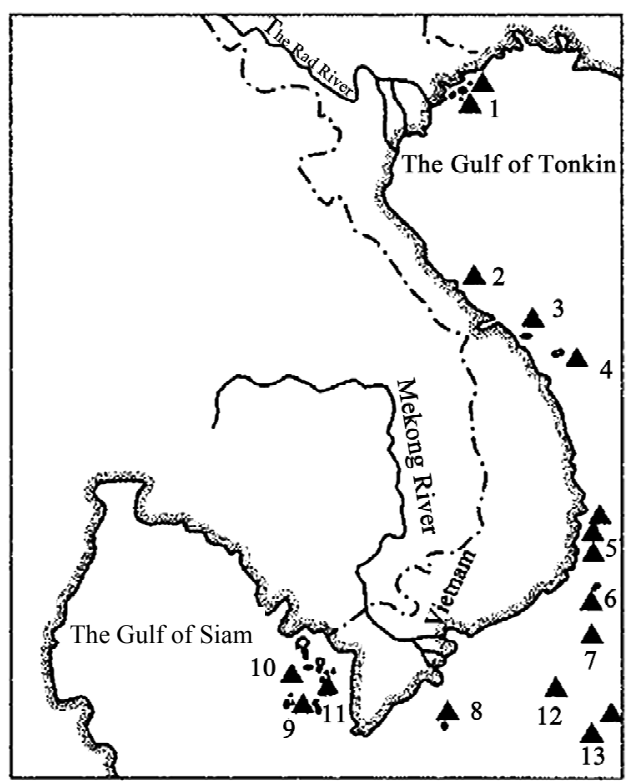

Figure 1. Schematized map of the surveyed regions. 1: Bai Tu Long Archipelago; 2: Ze Island; 3: Cape Danang, Cham and Son Tra islands; 4: Re Island; 5: reefs of the Khanh Hoa Province; 6: Thu Island; 7: Ca Thuik Islands; 8: Con Dao Islands; 9: Tho Chu Island; 10: An Thoi Archipelago and Namsu Islands; 11: Rach Gia bay; 12: Royal Bishop and Astrolab shoals; 13: Spratly Islands.

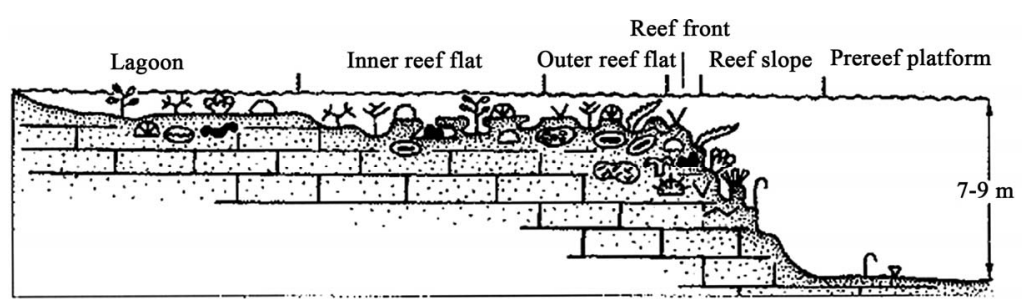

(a)

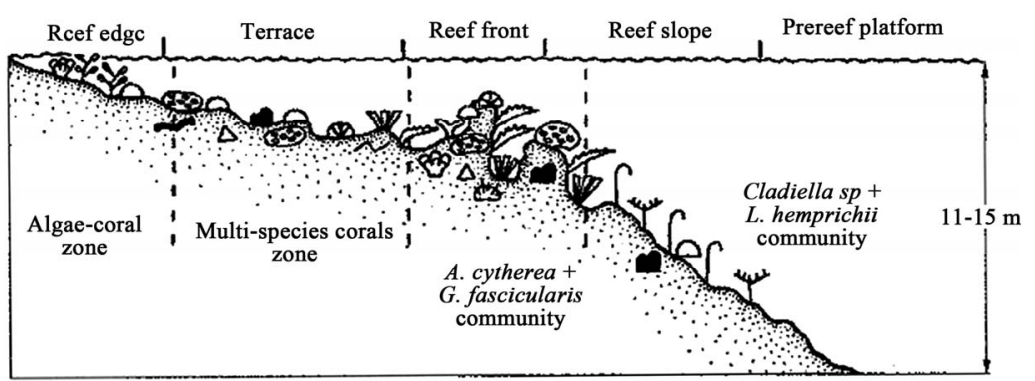

(b)

Figure 2. Schematized profiles of structural (a) and unstructured (b) reefs. See the text for comments. 
Reefs of the first type have a distinct zonation (reef lagoon, reef-flat, etc.) with a developed carbonate framework, so-called structural reefs [20] common for the tropical zone of the World Ocean. Reefs of the second type display a weak morphostructural zonation, with some zones occasionally absent. Carbonate deposits in such reefs comprise only coral settlements of a low thickness, hardly changing the overall substrate profile. These are so-called structureless [20] or encrusting [18] reefs. Vietnam's structural reefs are mainly formed in closed bights and on the organogenic base of Holocene reefs [21,22], while structureless reefs are formed off promontories and in open bights, mostly on stone and rocky substrates [18,23-25].

Vietnam's reefs pertain to the epicontinental monsoon type $[7,11,21,24,26,27]$. They are situated at the South China Sea periphery. The shoal waters of this region are highly eutrophicated, and the grounds are highly silted due to the huge amount of terrigenous influx. Other hydrological conditions are also not optimal for reef-building coral growth. Thus, in the Gulf of Tonkin, the salinity may drop to $26 \%$, and winter water temperature to $16^{\circ} \mathrm{C}$. Heavy northeast monsoon winds generate coastal waves up to three meters high with a 6-s period. During southwest monsoons, the Vietnam coast is struck by $5-10$ typhoons per year with the strongest storm excitements and strong desalination, that sometimes results in almost utter annihilation of coral reef [5,28]. All this renders appreciable influence on features of formation of reefs which occurs, as a rule, on a stony, rock-stony substratum and less often on coralogenous sediments of Holocene reefs. Vietnam's reefs feature a moderate vertical and horizontal spread and low thickness of modern reef-derived deposits. Their offshore spread usually does not exceed $200-300 \mathrm{~m}$. They rarely extend to a depth of over $20 \mathrm{~m}$. Sometimes they lack distinct morphological zonation. Most of Vietnam's reefs have an indistinct reef flat and slope. In some reefs, mostly ones on stone and boulder substrates, the only distinct zone is the reef slope. However, they all have a distinct vertical biological zonation, showing up in the dominant species succession and in the change in the composition and structure of coral communities and accompanying macrobenthos. Barrier, platform and fringing reefs on Holocene organogenous deposits always have well expressed morphological zonality, characteristic for the majority of reefs Indo-pacific [12, $27,28]$.

\section{Results}

\subsection{Species Diversity}

According to the studies performed in the first decades of the twenty first century, Vietnam's reef-building coral fauna comprises 350 species (see Table 1), pertaining to 80 genera (including 9 ahermatypic corals), of which 137 species, belonging to 26 genera, were not previously known for that region, and 13 species from 6 genera were described for the first time [11,18,29-33]). As in most Indo-Pacific reefs [5,19,24,34-36], the species diversity of Vietnam's reefs consists mainly of the members of 5 families, Acroporidae (117 species), Faviidae (42 species), Fungiidae (32 species), Poritidae (31 species), and Dendrophylliidae ( 25 species), making up altogether $64.48 \%$ of the total scleractinian species composition (Figure 3). The five genera most diverse and widespread in all reefs comprise Acropora (90 species), Montipora (28 species), Porites (20 species), Favia (14 species), and Fungia (12 species) are most various and numerous on all reefs, making $47 \%$ of all specific riches of scleractinian (Figure 4).

In all, some 20 scleractinian species form mono-specific settlements, varying from small "spots" (tens of square meters) to extended zones (hundreds of square meters), with a coverage reaching $60 \%-100 \%$. One fifth of all scleractinians occur throughout the Vietnam coast (Figure 5).

As a whole, the species diversity of reef-building scleractinians in different areas of the Vietnam coast is quite comparable, ranging from 190 species in the Gulf of Tonkin to 265 in the South Vietnam (Figure 6). Similar (193 - 256) numbers of species were reported for reefs of Indonesia, the Philippines, and Western Australia [36,37]. Central and South Vietnam reefs are most similar in species composition and are quite comparable to Spratly reefs). The degree of similarity of specific composition of Scleractinian various areas of Vietnam is resulted on the clustered diagram (Figure 7).

The peculiarity of the coral faunas of the Siam and Tonkin gulfs as revealed by cluster analysis (Figure 8) is consistent with their ecological peculiarities [7,23,38,39]. Their scleractinian diversity is partly caused by their similar hydrological regimes. Both gulfs are shallows with high water eutrophycation and turbidity, with a predominance of clay sediments.

These factors cause a similarity of the biological and morphostructural zonation of reefs and species composition of reef communities in the gulfs. At the same time, certain differences in climatic and geomorphological conditions of the gulfs result in some dissimilarities in their scleractinian species composition. The development, zonation, species composition, and structure of the reefs in the gulfs were reported previously $[13,23,39,40]$, so here, only major similarities and differences will be mentioned. 
Table 1. List species of scleractinian of vietnam.

\begin{tabular}{|c|c|c|c|c|c|c|c|}
\hline Species & $\begin{array}{l}\text { Gulf of } \\
\text { Tonkin }\end{array}$ & $\begin{array}{l}\text { Gulf of } \\
\text { Siam }\end{array}$ & $\begin{array}{c}\text { Central } \\
\text { Vietnam }\end{array}$ & $\begin{array}{c}\text { Nhatrang } \\
\text { Bay }\end{array}$ & $\begin{array}{c}\text { Island } \\
\text { Thu }\end{array}$ & $\begin{array}{l}\text { Kondao } \\
\text { Islands }\end{array}$ & $\begin{array}{c}\text { Spratly } \\
\text { archipelago }\end{array}$ \\
\hline Acantastrea echinata (Dana, 1846) & & + & + & + & & + & + \\
\hline Acantastrea hemprichii (Erhenberg, 1834) & & + & + & + & & & \\
\hline Acantastrea hillae (Wells, 1955) & & + & + & + & & & + \\
\hline Acropora abrolhosensis (Veron, 1985) & + & & + & & & & + \\
\hline Acropora abrotonoides (Lamarck, 1816) & & + & + & + & + & + & \\
\hline Acropora aculeus (Dana, 1846) & & & & & & + & + \\
\hline Acropora acuminata (Verril, 1864) & + & + & + & & & + & \\
\hline Acropora anthocercis (Brook, 1892) & & & & + & & + & \\
\hline Acropora aspera (Dana, 1846) & + & + & + & + & + & + & + \\
\hline Acropora austera (Dana, 1846) & + & + & & & & + & + \\
\hline Acropora brueggemanni (Brokk, 1893) & + & & + & & & + & \\
\hline Acropora cerealis (Dana, 1846) & + & & + & + & & + & + \\
\hline Acropora clathrata (Brook, 1981) & & & + & & + & & \\
\hline Acropora cytherea (Dana, 1846) & + & + & + & + & + & + & + \\
\hline Acropora dendrum (Bassett-Sminh, 1890) & & & & + & & & \\
\hline Acropora digitifera (Dana, 1846) & + & + & + & + & + & + & + \\
\hline Acropora divaricata (Dana, 1846) & + & + & + & + & + & + & + \\
\hline Acropora donei (Veron \& Wallace, 1984) & & & + & & & & \\
\hline Acropora elseyi (Brook, 1892) & & + & & & & + & \\
\hline Acropora fasciculare (Latypob, 1992) & & & + & & & & \\
\hline Acropora florida (Dana, 1846) & + & + & + & + & + & + & + \\
\hline Acropora formosa (Dana, 1846) & + & + & + & + & + & + & + \\
\hline Acropora gemmifera (Brok, 1892) & + & & + & + & + & & + \\
\hline Acropora glauca (Brook, 1893) & + & & + & & & & \\
\hline Acropora grandis (Brook, 1892) & + & + & + & + & + & + & + \\
\hline Acropora granulosa (Edw. \& Haime, 1860) & & + & + & + & + & + & + \\
\hline Acropora horrida (Dana, 1846) & & & & & + & & \\
\hline Acropora humilis (Dana, 1846) & + & + & + & + & + & + & + \\
\hline Acropora insignis (Nemenzo, 1967) & & + & & & & + & \\
\hline Acropora hyacinthus (Dana, 1846) & + & + & + & + & + & + & + \\
\hline Acropora latistella (Brook, 1892) & & & + & + & & & \\
\hline Acropora listeri (Brook, 1893) & & + & + & + & & + & \\
\hline Acropora longicyathus (Edw. \& Haime, 1860) & & + & + & & + & + & + \\
\hline Acropora loripes (Brook, 1892) & + & + & & + & & + & \\
\hline Acropora lutkeni (Crossland, 1952) & + & + & & + & & & \\
\hline Acropora microclados (Ehrenberg, 1834) & & + & + & & & & \\
\hline Acropora microphthalma (Verril, 1869) & & + & + & & + & + & + \\
\hline
\end{tabular}


Acropora millepora (Ehrenberg, 1834)

Acropora monticulosa (Brüggemann, 1879)

Acropora multiacuta (Nemenzo, 1967)

Acropora nasuta (Dana, 1846)

Acropora nobilis (Dana, 1846)

Acropora palmerae Wells, 1954

Acropora parilis (Quelch, 1886)

Acropora paniculata (Verrill, 1902)

Acropora papillare (Latypov, 1992)

Acropora pulchra (Brook, 1891)

Acropora pulchra (Brook, 1891)

Acropora robusta (Dana, 1846)

Acropora samoensis (Brook, 1891)

Acropora sarmentosa (Brook, 1891)

Acropora secale (Studer, 1878)

Acropora selago (Studer. 1878)

Acropora solitariensis (Veron \& Wallace,1984)

Acropora squamata (Latypov, 1992)

Acropora speciosa (Quelch, 1886)

Acropora subglabra (Brook, 1891)

Acropora tenuis (Dana, 1846)

Acropora valenciennesi (Edw. \& Haime, 1860)

Acropora valida (Dana, 1846)

Acropora vaughani (Wells, 1954)

Alveopora allingi (Hoffmeister, 1925)

Alveopora marionensis (Veron \& Pichon, 1982)

Alveopora verrilliana (Dana, 1872)

Anacropra forbesi (Ridley, 1884)

Astreopora cuculata (Lamberts, 1980)

Astreopora listeri (Bernard, 1896)

Astreopora longiseptata sp nov

Astreopora myriophthalma (Lamarck, 1816)

Astreopora ocellata (Bernard, 1896)

Australogyra zelli (Veron \& Pichon, 1977)

Balanophyllia cummingii (Edw. \& Haime, 1848)

Balanophyllia stimpsoni (Verrill, 1865)

Barabattoia amicorum (Edw. \& Haime, 1850)

Barabattoia mirabilis (Yabe \& Sugiyama, 1941)

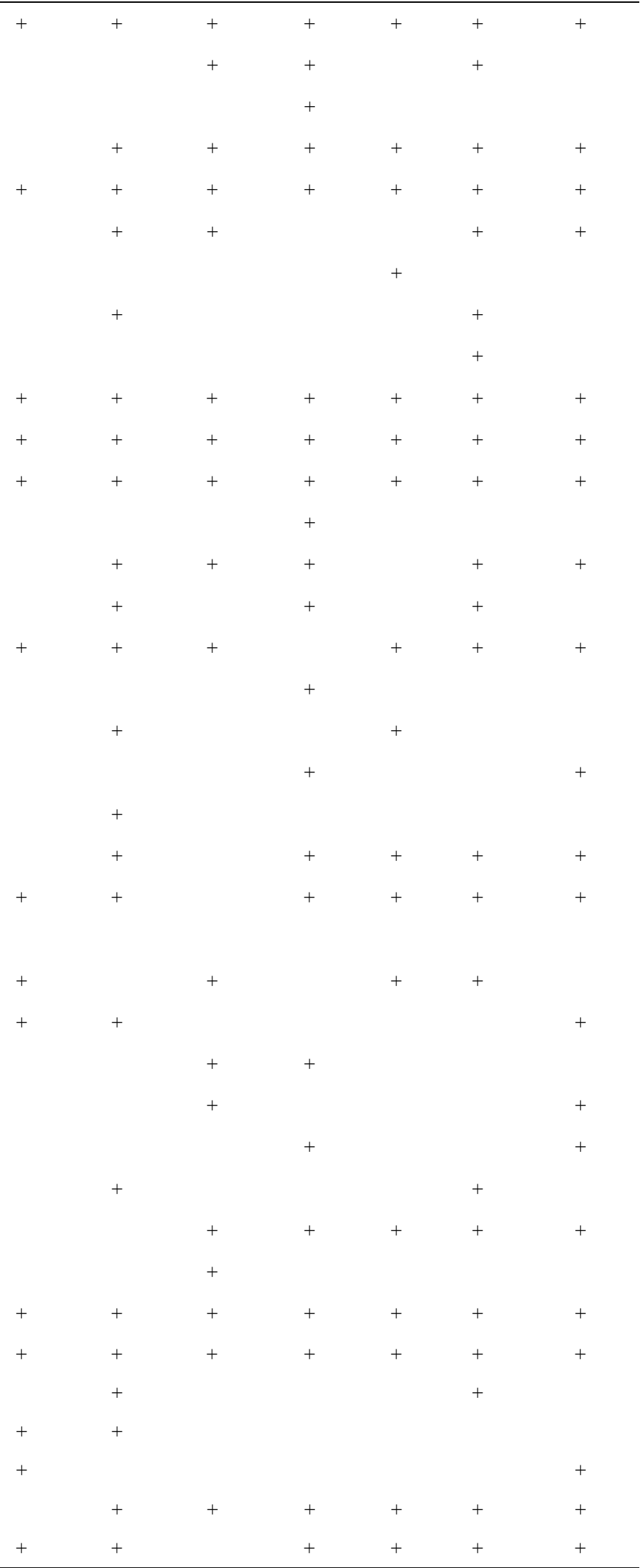


Caulastrea furcata (Dana, 1846)

Caulastrea tumida (Matthai, 1928)

Coeloseris mayeri (Vaughan, 1918)

Coscinaraea columna (Dana, 1846)

Coscinaraea exesa (Dana, 1846)

Coscinaraea mcneilli (Wells, 1962)

Ctenactis echinata (Pallas, 1766)

Cycloseris cf. Sinensis (Edw. \& Haime, 1851)

Cycloseris costulata (Ortman, 1889)

Cycloseris cyclolites (Lamarck, 1801)

Cycloseris densicolummelus sp. nov.

Cycloseris patelliformis (Boschma, 1923)

Cycloseris somervillei (Gardiner, 1909)

Cycloseris tenuis (Dana, 1846)

Cycloseris vaughani (Boschma, 1923)

Cynarina lacrymalis (Edw. \& Haime, 1848)

Cyphastrea chalcidicum (Forskål, 1775)

Cyphastrea japonica (Yabe \& Sugiyama, 1932)

Cyphastrea microphthalma (Lamarck, 1816)

Cyphastrea serailia (Forskål, 1775)

Dendrophyllia aculeata sp. nov.

Dendrophyllia arbuscula (Van der Horst, 1922)

Dendrophyllia cornigera (Lamarck, 1816)

Dendrophyllia horsti (Gard. \& Waugh, 1939)

Dendrophyllia japonica (Regberg, 1892)

Dendrophyllia laborelli (Zibr. \& Brilo, 1984)

Dendrophyllia robusta (Bourne, 1905)

Dendrophyllia sphaerica (Nemenzo, 1981)

Diaseris fragilis (Alcock, 1893)

Diploastrea heliopora (Lamarck, 1816)

Echinophyllia aspera (Ellis \& Solander, 1786)

Echinophyllia echinata (Saville-Kent, 1975)

E. echinoporoides (Veron \& Pichon, 1980)

Echinophyllia nichihirai (Veron \& Pichon, 1990)

\begin{tabular}{|c|c|c|c|c|c|c|}
\hline & + & & + & & + & \\
\hline & + & + & + & & & + \\
\hline & + & + & & & & + \\
\hline+ & + & + & + & + & + & + \\
\hline & + & + & + & & & \\
\hline & & + & & & & \\
\hline & + & + & + & + & + & + \\
\hline & + & + & & & & + \\
\hline & + & + & + & & + & \\
\hline & + & + & + & & + & + \\
\hline & + & & & & & \\
\hline & + & + & & & & + \\
\hline & & & & & + & \\
\hline & & + & & & + & + \\
\hline & & + & & & + & + \\
\hline+ & + & & + & & & + \\
\hline+ & + & + & + & & & + \\
\hline & + & & + & & & \\
\hline & + & + & + & + & + & + \\
\hline+ & + & + & + & + & + & + \\
\hline+ & & & & & + & \\
\hline+ & & + & & & + & \\
\hline & & & & & & + \\
\hline+ & & + & & & + & \\
\hline & + & & & & + & \\
\hline+ & & & & & & \\
\hline & & & + & & & + \\
\hline+ & + & & & & & \\
\hline & + & + & + & & & + \\
\hline+ & + & + & + & + & + & + \\
\hline+ & + & + & + & & & + \\
\hline+ & + & + & & & + & \\
\hline & + & + & + & + & + & + \\
\hline & + & & + & & & + \\
\hline
\end{tabular}


Echinophyllia orpheensis (Veron \& Pichon, 1979)

Echinophyllia patula (Hodgson \& Ross, 1981)

Echinopora gemmacea (Lamarck, 1816)

Echinopora hirsutissima (Edw. \& Haime, 1849)

Echinopora horrida (Dana, 1846)

Euphyllia ancora (Veron \& Pichon, 1980)

Euphyllia cristata (Chevalier, 1971)

Euphyllia divisa (Veron \& Pichon, 1980)

E. glabrescens (Chamisso \& Eysenhard, 1821)

Euphyllia grandiseptata sp. nov.

Euphyllia yaeyamaensis (Shirai, 1980)

Favia favus (Forskål, 1775)

Favia laxa (Klunzinger, 1879)

Favia lizardensis (Veron \& Pichon, 1977)

Favia maritima (Nemenzo, 1971)

Favia matthai (Vaughan, 1918)

Favia maxima (Veron et al., 1977)

Favia pallida (Dana, 1846)

Favia rotumana (Gardiner, 1889)

Favia rotundata (Veron \& Pichon, 1977)

Favia speciosa (Dana, 1846)

Favia stelligera (Dana, 1846)

Favia truncatus (Veron, 2000)

Favia veroni (Moll \& Best, 1984)

Favites abdita (Ellis \& Solander, 1786)

Favites chinensis (Verrill, 1866)

Favites complanata (Ehrenberg, 1834)

Favites flexuosa (Dana, 1846)

Favites halicora (Ehrenberg, 1834)

Favites pentagona (Esper, 1794)

Favites russelli (Wells, 1954)

Favites solidocolumellae sp. nov.

Favites virens (Dana, 1846)

Fungia concina (Verrill, 1864)

$+$ 
Fungia corona (Döderlein, 1901)

Fungia danai (Edwards \& Haime, 1851)

Fungia fungites (Linnaeus, 1758)

Fungia granulosa (Klunzinger, 1879)

Fungia horrida (Dana, 1846)

Fungia repanda (Dana, 1846)

Fungia scabra (Döderlein, 1901)

Fungia scruposa (Klunzinger, 1879)

Fungia scutaria (Lamarck, 1801)

Fungia seychelensis (Hoeksema, 1993)

Fungia valida (Verrill, 1864)

Galaxea astreata (Lamarck, 1816)

Galaxea crassiseptata (Latypov, 1998)

Galaxea fascicularis (Linnaeus, 1767)

Galaxea vesiculosa sp. nov.

Gardineroseris pavonoides sp. nov.

Gardineroseris planulata (Dana, 1846)

Goniastrea aspera (Verrill, 1865)

Goniastrea australiensis (Edw. \& Haime, 1857)

Goniastrea cf. palauensis (Yabe et al., 1936)

Goniastrea edwardsi (Chevalier, 1971)

Goniastrea favulus (Dana, 1846)

Goniastrea pectinata (Ehrenberg, 1834)

Goniastrea retiformis (Lamarck, 1816)

Goniopora columna (Dana, 1846)

Goniopora djiboutiensis (Vaughan, 1907)

Goniopora fruticosa (Saville-Kent, 1893)

Goniopora lobata (Edwards \& Haime, 1851)

Goniopora pandoraensis (Veron \& Pichon, 1982)

Goniopora stokesi (Edwards \& Haime, 1851)

Goniopora stutchburyi (Wells, 1955)

Goniopora tenuidens (Quelch, 1886)

Halomitra pileus (Linnaeus, 1758)

Heliofungia actiniformis (Quoy \& Gaimard,1833)

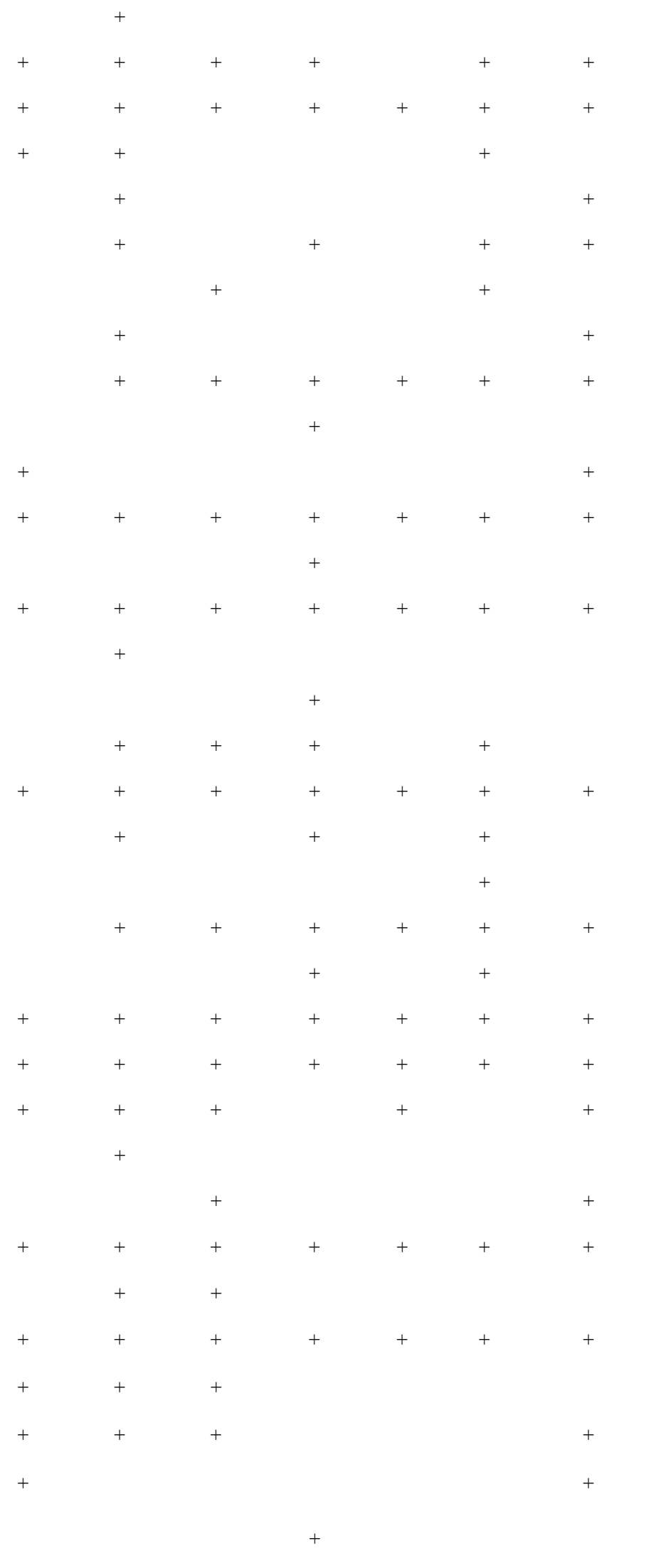


Herpolitha limax (Esper, 1797)

Herpolitha weberi (Van der Horst, 1921)

Heterocyathis aequicostatus (Edw. \& Haime,1848)

Heteropsammia cochlea (Spengler, 1781)

Hydnophora exesa (Pallas, 1766)

Hydnophora microconos (Lamarck, 1816)

Hydnophora rigida (Dana, 1846)

Isopora cuneata (Dana, 1846)

Isopora palifera (Lamarck, 1816)

Leptastrea bottae (Edwards \& Haime, 1849)

Leptastrea pruinosa (Crossland, 1952)

Leptastrea purpurea (Dana, 1846)

Leptastrea transversa (Klunzinger, 1879)

Leptoria phrygia (Ellis \& Solander, 1786)

Leptoseris explanata (Yabe \& Sugiyama, 1941)

Leptoseris gardineri (Van der Horst, 1921)

Leptoseris hawaiiensis (Vaughan, 1907)

Leptoseris mycetoseroides (Wells, 1954)

Leptoseris scaba (Vaughan, 1907)

Leptoseris var.mycetoseroides (Wells, 1954)

Leptoseris yabei (Pillai \& Scheer, 1976)

Lithophyllon bistomatum (Latypov, 1995)

Lithophyllon mokai (Hoeksema, 1989)

Lithophyllon undulatum (Rehberg, 1892)

Lobophyllia grandis sp. nov.

Lobophyllia corymbosa (Foskål, 1775)

Lobophyllia flabellioformis (Veron, 2000)

L. hattai (Yabe, Sygiyama \& Eguchi, 1936)

Lobophyllia hemprichii (Ehrenberg, 1834)

Lobophyllia pachysepta (Chevalier, 1975)

Lobophyllia robusta (Yabe \& Sugiyama, 1936)

Madracis kirbyi (Veron \& Pichon, 1976)

Merulina ampliata (Ellis \& Solander, 1786)

Merulina scabriscula (Dana, 1846)

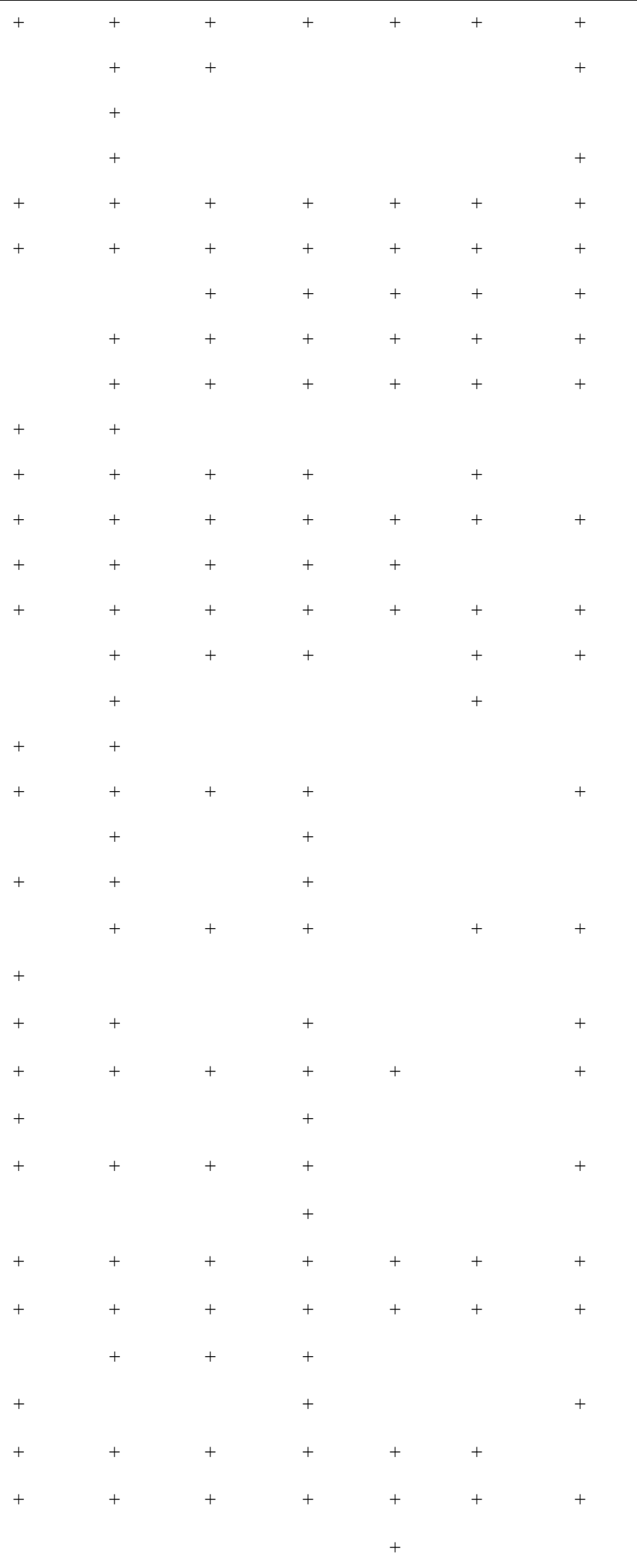


Montastrea annuligera (Edw. \& Haime, 1849)

Montastrea colemani (Veron, 2000)

Montastrea curta (Dana, 1846)

Montastrea magnistellata (Chevalier, 1971)

Montastrea valenciennesi (Edw. \& Haime, 1848)

Montipora aequituberculata (Bernard, 1897)

Montipora angulata (Lamarck, 1816)

Montipora australiensis (Bernard, 1897)

Montipora caliculata (Dana, 1846)

Montipora crassituberlata (Bernard, 1897)

Montipora danae (Edwards \& Haime, 1851)

Montipora digitata (Dana, 1846)

Montipora efflorescens (Bernard, 1897)

Montipora foliosa (Pallas, 1766)

Montipora grisea (Bernard, 1897

Montipora hispida (Dana, 1846)

Montipora hoffmeisteri (Wells, 1954)

Montipora informis (Bernard, 1897)

Montipora millepora (Crossland, 1952)

Montipora molis (Bernard, 1897)

Montipora monasteriata (Forskål, 1775)

Montipora nodosa (Dana, 1846)

Montipora porites (Veron, 2000)

Montipora spongodes (Bernard, 1897)

Montipora spumosa (Lamarck, 1816)

Montipora stellata (Bernard, 1897)

Montipora tuberculosa (Lamarck, 1816)

Montipora turgescens (Bernard, 1897)

Montipora turtlensis (Veron \& Wallace, 1984)

Montipora undata (Bernard, 1897)

Montipora venosa (Ehrenberg, 1834)

Montipora verrucosa (Lamarck, 1816)

Montipora vietnamensis (Veron, 2000)

Moseleya latistellata (Quelch, 1884)

\begin{tabular}{|c|c|c|c|c|c|c|}
\hline+ & + & + & $\begin{array}{l}+ \\
+\end{array}$ & & + & + \\
\hline+ & + & + & + & & + & + \\
\hline+ & & & & & & \\
\hline+ & + & + & + & & + & + \\
\hline+ & + & + & + & + & + & + \\
\hline & & + & + & & + & \\
\hline+ & + & & & & + & + \\
\hline+ & + & & + & & + & + \\
\hline & & + & + & + & + & + \\
\hline+ & & + & & & + & \\
\hline+ & + & + & + & + & + & + \\
\hline & + & & & & & + \\
\hline & + & & + & & & + \\
\hline & + & & + & + & & + \\
\hline+ & + & + & + & + & + & + \\
\hline & + & + & + & & + & \\
\hline & + & & & & & \\
\hline & + & + & + & + & & + \\
\hline & & & & & + & \\
\hline+ & & + & & & & + \\
\hline+ & + & + & + & & & \\
\hline & & + & & & & + \\
\hline+ & + & + & + & & + & + \\
\hline+ & + & & & & & + \\
\hline & + & + & & & & \\
\hline+ & + & + & + & + & + & + \\
\hline+ & + & + & + & + & + & + \\
\hline+ & & & + & & & \\
\hline+ & + & + & + & & + & + \\
\hline+ & + & & & & + & \\
\hline & + & + & + & + & + & + \\
\hline & & & + & & & \\
\hline & + & & & & & + \\
\hline
\end{tabular}


Mycedium elephantotus (Pallas, 1766)

$+$

$+$

Oulastrea alta (Nemenzo, 1981)

Oulastrea crispata (Lamarck, 1816)

Oulophyllia bennettae (Veron \& Pichon, 1977)

Oulophyllia crispa (Lamarck, 1816)

Oulophyllia levis (Nemenzo, 1959)

Oxypora glabra (Nemenzo, 1959)

Oxypora lacera (Verrill, 1864)

Pachyseris rugosa (Lamarck, 1801)

Pachyseris speciosa (Dana, 1846)

Pachyseris monticulosa sp. nov.

Palauastrea ramosa (Yabe \& Sugiyama, 1941)

Pavona cactus (Forskål, 1775)

Pavona clavus (Dana, 1846)

Pavona decussata (Dana, 1846)

Pavona distincta sp. nov.

Pavona explanulata (Lamarck, 1816)

Pavona frondifera (Lamarck, 1801)

Pavona maldivensis (Gardiner, 1905)

Pavona minuta (Wells, 1954)

Pavona varians (Verrill, 1864)

Pavona venosa (Ehrenberg, 1834)

Pectinia alcicornis (Saville-Kent, 1871)

Pectinia lactuca (Pallas, 1766)

Pectinia paeonia (Dana, 1846)

Physogyra lichtensteini (Edw. \& Haime, 1851)

Platygyra daedalia (Ellis \& Solander, 1786)

Platygyra lamellina (Ehrenberg, 1834)

Platygyra pini (Chevalier, 1975)

Platygyra sinensis (Edw. \& Haime, 1849)

Plerogyra sinuosa (Dana, 1846)

Plesiastrea versipora (Lamarck, 1816)

Pleuractis moluccensis (Van der Horst, 1919)

Pleuractis paumotensis (Stuthbury, 1833) 
Pocillopora capitata (Verrill, 1864)

Pocillopora damicornis (Linnaeus, 1758)

Pocillopora eydouxi (Edwards \& Haime, 1860)

Pocillopora kelleheri (Veron, 2000)

Pocillopora meandrina (Dana, 1846)

Pocillopora verrucosa (Ellis \& Solander)

Pocillopora woodjonesi (Vaughan, 1918)

Podabacia crustacea (Pallas, 1766)

Polyphyllia novaehiberniae (Lesson, 1831)

Polyphyllia talpina (Lamarck, 1831)

Porites annae (Crossland, 1952)

Porites attenuata (Nemenzo, 1955)

Porites australiensis (Vaughan, 1918)

Porites cylindrica (Dana, 1846)

Porites deformis (Nemenzo, 1955)

Porites densa (Vaughan, 1918)

Porites lichen (Dana, 1846)

Porites lobata (Dana, 1846)

Porites lutea (Edwards \& Haime, 1860)

Porites mayeri (Vaughan, 1918)

Porites monticulosa (Dana, 1846)

Porites mordax (Dana, 1846)

Porites murrayensis (Vaughan, 1918)

Porites nigrescens (Dana, 1848)

Porites rus (Forskål, 1775)

Porites solida (Forskål, 1775)

Porites $s p .1$

Porites $s p .2$

Porites stephensoni (Crossland, 1952)

Porites vaughani (Crossland, 1952)

Psammocora contigua (Esper, 1979)

Psammocora digitata (Edwards \& Haime, 1851)

Psammocora explanulata (Van der Horst, 1922)

Psammocora nierstraszi (Van der Horst, 1921) 
Psammocora profundacella (Gardiner, 1898)

Psammocora superficialis (Gardiner, 1898)

Pseudosiderastrea tayamai (Yabe \& Sug., 1935)

Sandalolitha dentata (Quelch, 1884)

Sandalolitha robusta (Quelch, 1886)

Scolymia aff. Vitiensis (Brüggemann, 1877)

Scolymia australis (Edw. \& Haime, 1849)

Seriatopora caliendrum (Ehrenberg, 1834)

Seriatopora hystrix (Dana, 1846)

Stylocoeniella guentheri (Basset-Smith, 1890)

Stylophora pistillata (Esper, 1797)

Stylophora subseriata (Ehrenberg, 1834)

Symphyllia agaricia (Edwards \& Haime, 1849)

Symphyllia erythraea (Klunzinger, 1879)

Symphyllia hassi (Pillai \& Scheer, 1976)

Symphyllia radians (Edwards \& Haime, 1849)

Symphyllia recta (Dana, 1846)

Symphyllia valenciennesii (Edw. \& Haime, 1849)

Trachyphyllia geoffroyi

Tubastrea aurea (Quoy \& Gaimard, 1833)

Tubastrea coccinea (Ehrenberg, 1834)

Tubastrea diaphana (Dana, 1846)

Tubastrea micrantha (Ehrenberg, 1834)

Turbinaria bifrons (Brüggemann, 1877)

Turbinaria conspicua (Bernard, 1896)

Turbinaria contorta (Bernard, 1896)

Turbinaria crater (Pallas, 1766)

Turbinaria frondens (Dana, 1846)

Turbinaria mesenterina (Dana, 1846)

Turbinaria patula (Dana, 1846)

Turbinaria peltata (Esper, 1794)

Turbinaria radicalis (Bernard, 1896)

Turbinaria reniformis (Bernard, 1896)

Turbinaria stellulata (Lamarck, 1816)

\begin{tabular}{|c|c|c|c|c|c|c|}
\hline+ & + & + & + & + & + & \\
\hline+ & + & + & & & + & + \\
\hline+ & + & + & + & + & + & + \\
\hline+ & + & + & + & + & + & + \\
\hline \multirow[t]{5}{*}{+} & + & + & + & + & + & + \\
\hline & + & + & + & & + & \\
\hline & + & & + & + & + & + \\
\hline & + & & & & & \\
\hline & + & + & + & + & + & + \\
\hline+ & & & + & + & + & + \\
\hline \multirow[t]{2}{*}{+} & + & + & + & + & + & + \\
\hline & + & & & + & & \\
\hline \multirow[t]{2}{*}{+} & + & & + & & + & + \\
\hline & & & + & & & \\
\hline+ & + & & & & & + \\
\hline+ & + & + & + & + & + & + \\
\hline+ & + & + & + & + & + & + \\
\hline \multirow[t]{2}{*}{+} & + & + & + & + & + & + \\
\hline & & & + & + & + & + \\
\hline+ & + & + & + & + & + & + \\
\hline \multirow[t]{2}{*}{+} & + & & + & + & + & + \\
\hline & & & + & + & + & \\
\hline+ & + & + & + & + & + & + \\
\hline \multirow[t]{3}{*}{+} & + & + & + & + & + & \\
\hline & + & + & & & & + \\
\hline & + & & + & & & \\
\hline+ & + & & & & & + \\
\hline+ & + & + & + & + & + & + \\
\hline \multirow[t]{2}{*}{+} & + & + & + & + & + & + \\
\hline & + & + & + & & & \\
\hline+ & + & + & + & + & + & + \\
\hline+ & + & & + & & & + \\
\hline+ & + & + & + & + & + & + \\
\hline & + & & & & & \\
\hline
\end{tabular}




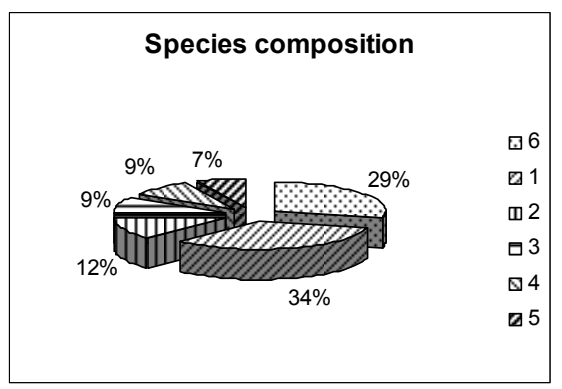

Figure 3. Relative species diversity of major coral genera in Vietnam. 1: Acroporidae; 2: Faviidae; 3: Fungiidae; 4: Poritidae; 5: Dendrophillidae; 6: The rest genera.

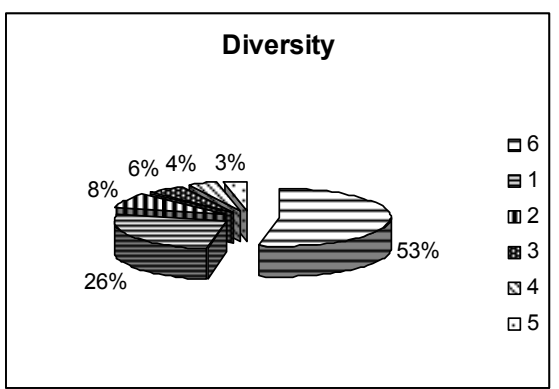

Figure 4. Reative species diversity of major coral species in Vietnam. 1: Acropora; 2: Montipora; 3: Porites; 4: Faia; 5: Fungia; 6: The res species.

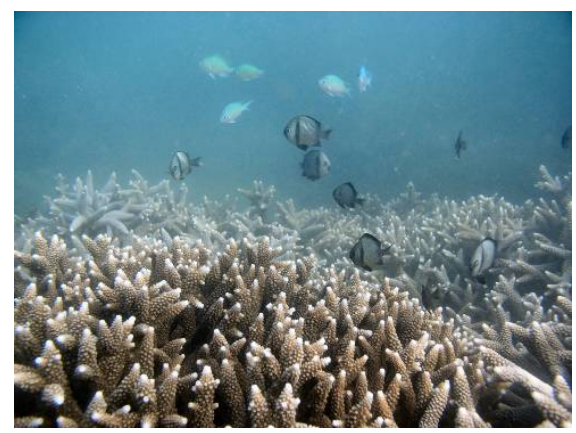

Figure 5. Monospecific settlement on reef Myeu Island at Nhatrang Bay, depth 2 m.

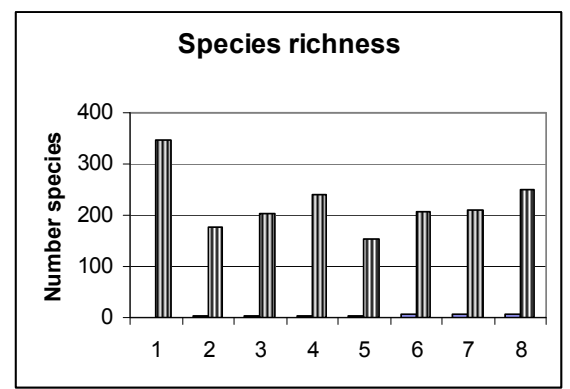

Figure 6. Scleractinian species diversity in different regions of Vietnam. 1: total number of species (347); 2: Gulf of Tonkin (177); 3: Central Vietnam (204); 4: South Vietnam (240); 5: Thu Island; 6: Con Dao Islands (211); 7: Spratly Island (211), 8: Gulf of Siam (251).

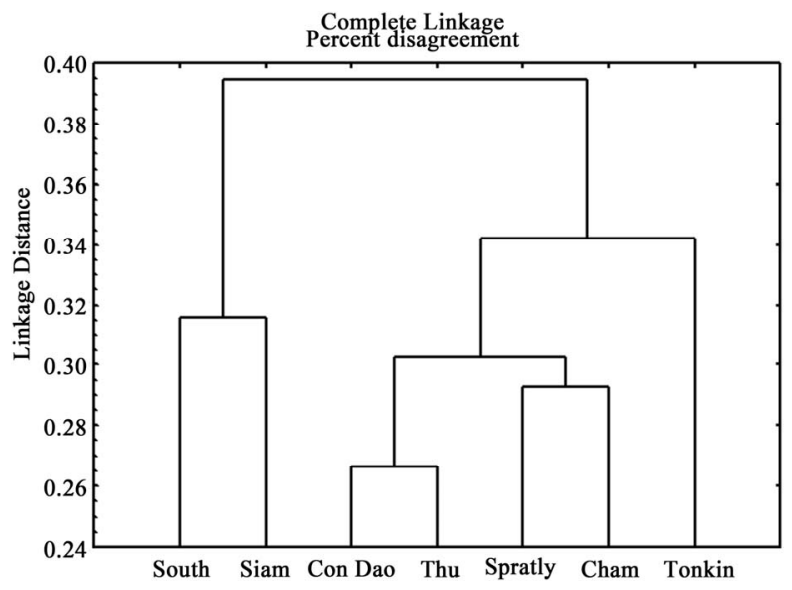

Figure 7. Cluster analysis of similarity species composition on different regions of Vietnam.

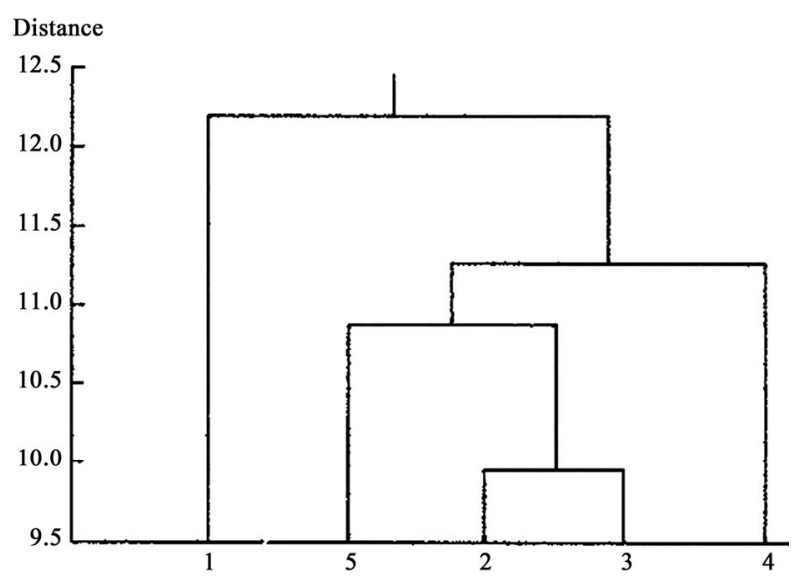

Figure 8. Similarity dendrogram of scleractinians faunas in different regions of Vietnam. 1: Gulf of Tonkin; 2: Central Vietnam; 3: South Vietnam; 4: Gulf of Siam; 5: Spratly Island.

The reef communities in both gulfs lack members of Palauastrea and Caulastrea and Acropora cuneata, the latter occurring in most Vietnam reefs. Plerogyra and Physogyra are absent in the closed part of the Gulf of Tonkin, and Pachyseris, Mycedium, and Pectinia, in the innermost and coastal areas of the Gulf of Siam. However, some members of the latter three genera and rare Physogyra and Plerogyra species are found in the open parts of both gulfs, off Hainan and Tho Chu islands. Corals having large polyp forms and capable of self cleaning-Galaxea, Echinopora, Lobophyllia, Echinophyllia, Turbinaria, Podobacia, Lithophyllon, Fungia, and Goniopora - are widespread in both gulfs. The reefs in both gulfs are dominated by many species of these genera (Galaxea fascicularis, Goniopora stokesi, Echinopora lamellosa, and Lobophyllia hemprichii), as well as by Acropora cytherea, A. nobilis, Montipora hispida, Porites lobata, and P. cylindrica, widespread in Indo- 
Pacific reefs. Altogether, these species cover $60 \%-80 \%$ of the substrate. Massive Porites colonies (at least 10 species) forming vast monospecific settlements are typical of both gulfs. At the same time, members of Pocillopora, abundant in most Indo-Pacific reefs (5-7 species), only rarely occur in the innermost parts of the gulfs (2 species maximum) but are common for island reefs in the open parts of the gulfs (Tho Chu, Hainan). By and large, the two gulfs are quite similar in coral diversity and share $74.3 \%$ of species.

\subsection{Communities}

The distribution and peculiarities of benthic communities in the coastal part of Vietnam reefs is rather constant (See Figure 2). As a rule, these are algal-coral communities, composed of several biocenoses (zones, facieses), dominated by individual algal or coral species or by groups of species (Figure 9). The predominance of Laurencia, Turbinaria, and Sargassum algae in the coastal zones of the reefs has been reported for many reef development areas. This may be indicative of an increase in water eutrophication or later stages of reef development [38,41-43].

Both along the Vietnam coast and in the whole IndoPacific, in reef zones characterized by relatively stable conditions (lagoons, deep stony and coral terraces, and reef slopes), branched, plate, and trumpet colonies of $A$. cytherea, A. hyacinthus, Montipora danae, M. foliosa, Porites cylindrica, P. nigrescens, and others successfully compete with differently shaped scleractinian colonies [43-45].

A wider distribution of encrusting and plate colonies of Euphyllia, Echinophyllia, Mycedium, Pachyseris, and Turbinaria compared to that of branched forms is directly caused by lowered illumination. This is also the case in many reefs of the Indo-Pacific and Caribbean basins [46-48]. In Vietnam's reefs, such corals are common for communities of the slope base, byoherms zone, and fore reef platform.

Caused by abiotic factors, the vertical distribution of reef-building corals has a strong effect on the development of biotic zonation across a whole reef community, beginning with settlement-site choice and ending with interspecific trophic relationships. The relationships between the species composition of benthic communities of some reefs as revealed by cluster analysis correlated with the ecological and physiographical zonation of the reefs (Figure 10).

Algal-coral lagoon and reef flat communities dominated by red and brown algae and similar in coral and common macrobenthos species composition formed a single cluster group, that of communities developing under similar conditions. The high similarity between coral faunas from different sites reflected similar, sometimes extreme, conditions of reef flat and shallow-water stone terrace. At the same time, communities of these reef zones were set apart from those of neighboring reef zones. Both in structural and unstructured reefs, polyspecific reef slope communities sharing a relatively greater number of corals also formed a distinct cluster. Polyspecific coral communities of a reef slope of structural and unstructured reefs distinctly stands apart on the greatest level of similarity of their specific variety.

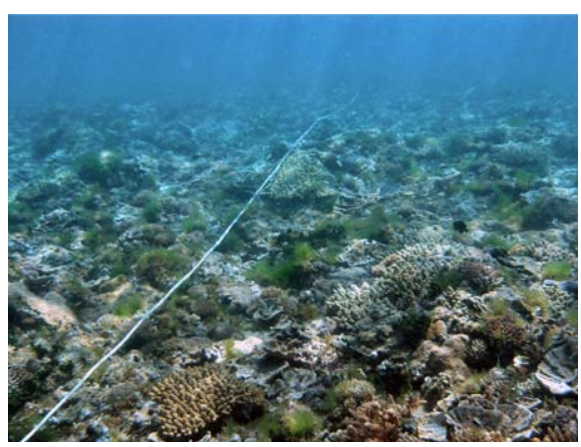

Figure 9. Algal-coral community on reef Bathlongvi, depth $2 \mathrm{~m}$.

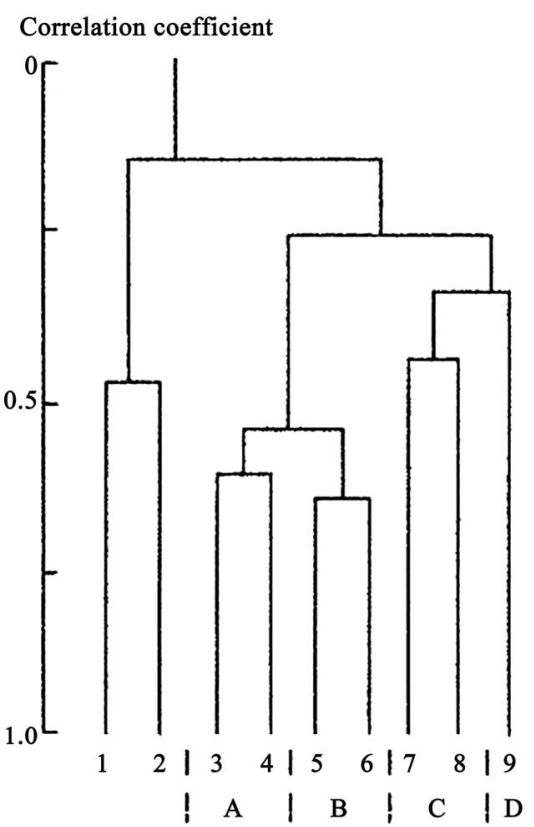

Figure 10. Cluster dendrogram of the species composition of benthic communities. Algal-coral community, unstructured (1) and structural (2) reefs; Acropora community, unstructured (3) and structural (4) reefs; Acropora + Diploastrea community, unstructured (5) and structural (6) reefs; (7) bioherm community (reef slope); (8) Junceella + Diaseris community; (9) Maleus + Junceella community. A: lagoon, $B$ : reef flat and terrace zone, $C$ : reef slope, D: for-reef platform. 


\section{Discussion and Conclusions}

To summarize the above, both structural and unstructured reefs feature vertical biological and geomorphological zonations. The latter is mainly determined by peculiarities of the underwater reef slope substrate. Similar biological zonation reflecting interzonal differences in environmental conditions (substrate, wave regime, sedimentation rate, illumination) has been reported for many of Vietnam's reefs and various Pacific and Caribbean reefs $[21,23,40,42,43]$.

Shallow-water Vietnam reefs growing in highly eutrophic conditions lack thick reef deposits $[18,23]$ and feature high coral diversity and distinct biological zonation, that is, the presence of inner heterotrophic (lagoon, reef flat) and outer autotrophic (reef slope) zones $[26,49,50]$, which is characteristic of typical Indo-Pacific reefs.

In reefs of Indonesia and Philippines and in the Great Barrier Reef, a total of 360 - 410 reef-building scleractinians pertaining to $70-80$ genera have been recorded [36]. This region of the Western Pacific is considered the center of origin of tropical coral faunas. The maximum coral diversity is observed in the so-called Coral Triangle $[36,51,52]$ with apices in the Philippines, the Malacca Peninsula, and New Guinea (Figure 11). To same fertile the center should expense and coast of Vietnam, scleractinian fauna which totals 350 species belonging to 80 genera.

Vietnam's reefs, too, obviously belong to this center, which is evidenced by their high similarity in coral species composition to reefs of Thailand, Indonesia, and the Philippines $(76.4 \%, 72.3 \%$, and $81.6 \%$, respectively). In the greater Western Pacific Coral Triangle (with apices in Vietnam, South Japan, and the Great Barrier Reef), coral faunas are also highly similar and homogenous. The similarities between the Vietnam coral fauna and those of Japan and Australia are $77.5 \%$ and $86 \%$, respectively, suggesting homogeneity of the coral fauna of the Western and Southwest Pacific. As a whole, the species complex of Vietnam scleractinians, as well as those of alcyonarians and gorgonarians, belongs to the tropical fauna as the majority of Vietnam corals are also common for the equatorial Indo-Pacific reef zone. The scleractinian species composition of this area exceeds $80 \%$ of that of the Pacific, and the alcyonarian diversity of Vietnam's reefs is one of the greatest in the Indo-Pacific $[18,31,53]$.

The species composition and high diversity of Vietnam's coral fauna, as well as its close similarity to the Southwest Pacific coral fauna, allow one to refer it to the Indonesia-Polynesian center of origin of the coral faunas of the tropical Indo-Pacific (Figure 12) The whole Vietnam coast, from the Gulf of Tonkin to the Gulf of Siam, is a biogeographically single whole and is part of the Indo-Polynesian Province of the Indo-Pacific Area.

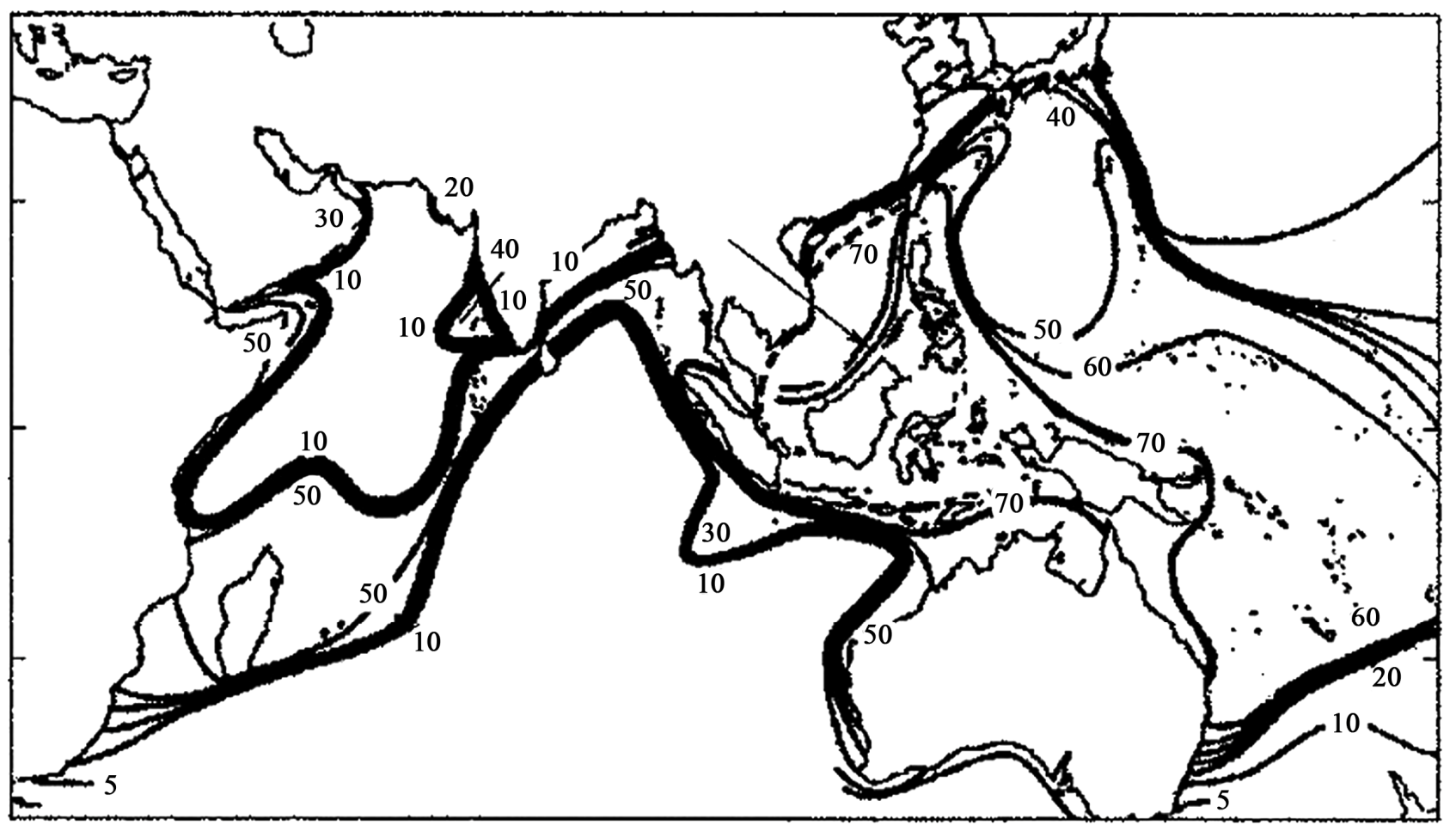

Figure 11. Schematized map of the generic diversity of reef-building corals in different regions of the Indo-Pacific (partly after Veron, 1995). The dotted line and arrow indicate new and old 70 genera diversity isolines, respectively. 


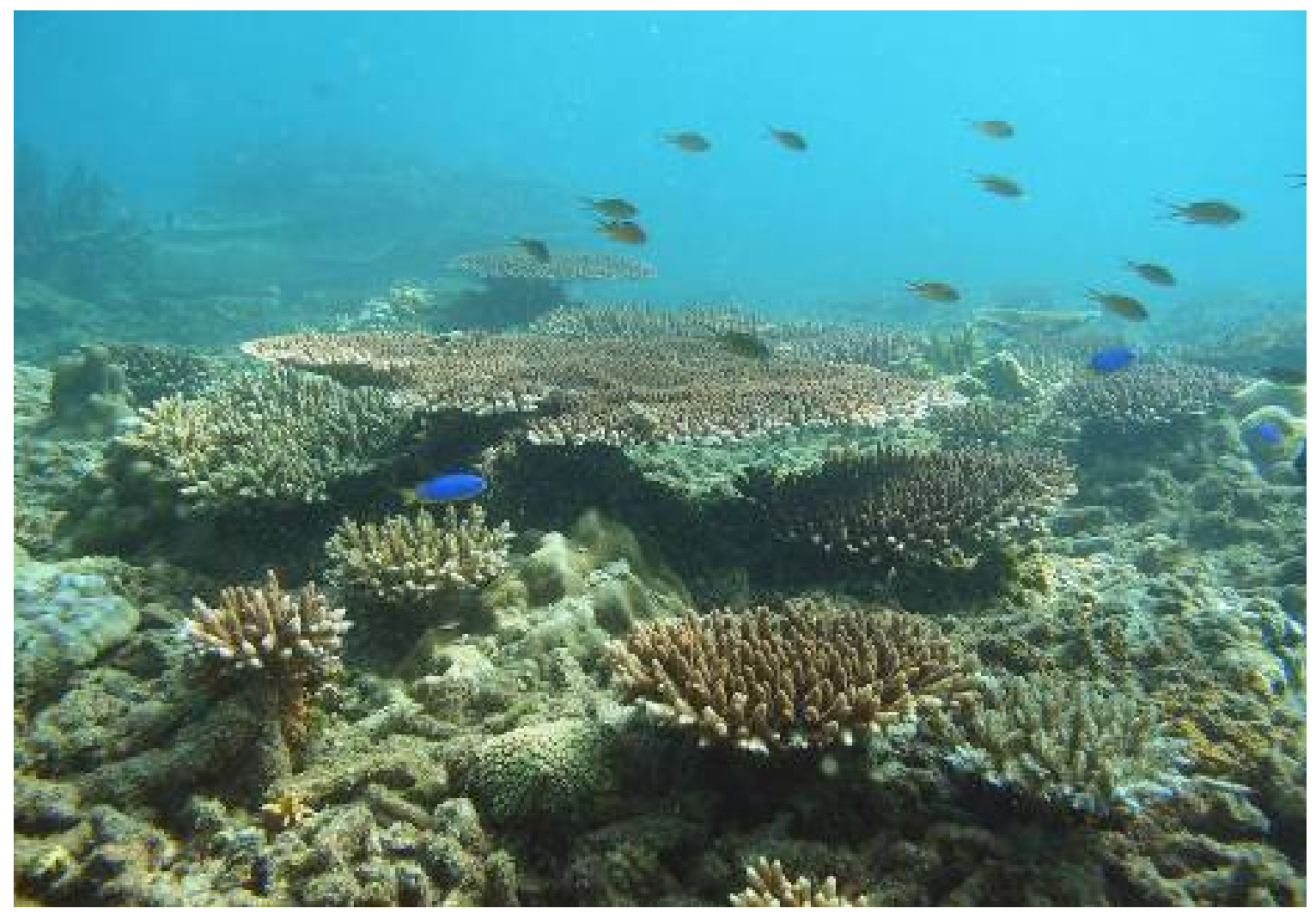

Figure 12. Optimal Vietnamese coral reef.

\section{Acknowledgements}

The author is sincerely grateful to the domestic and foreign colleagues V. Brykov, I. Budin, N. Selin, Yu. Yakovlev, Lang Van Ken, Нгуен Van Tien, Vo Si Tuan, assisting to me in researches and sent prints of their publications, special gratitude to Dr. Douglas Fenner for editing of the English text.

\section{References}

[1] T. D. Thanh, "Change in Environment and Ecosystems Relative to the Land-Sea Ineraction in the Vietnam Coastal Zone," EALOICZ Worshop, Qingdao, 1999, pp. $1-10$.

[2] Y. I. Sorokin, "Some Issues of Productivity, Trophology, and Energy Balance of a Coral Reef Ecosystem," Biologia Morya, No. 6, 1986, pp. 3-14.

[3] N. T. An, "Biological Productivity of Vietnam Marine, Waters, Monography on Vietnam Seas," Science and Technology Publishing Housing, Ha Noi, 1994, pp. 502-518.

[4] T. N. Dautova, Y. Y. Latypov and S. S. Dautov, "Composition and Structure of the Coral Communities of the Bai Ty Long Archipelago under Different Ecological Conditions," Biologia Morya, Vol. 25, No. 2, 1999, pp. 106-107.
[5] S. T. Vo and G. Hodgson, "Coral Reefs of Vietnam: Recruitment Limitation and Physical Forcing," Proceedings of 8th International Coral Reef Symposium, Vol. 1, 1997, pp. 477-482.

[6] R. Sérene, "Inventaires des Invertebres Marine de l'Indochine," Institute of Oceanography Indochine, Vol. 30, 1937, pp. 3-83.

[7] C. Dawydoff, "Contribution et l'Etude des Invertebres de la Faune Marine Benthique de l'Indochina," Buletin Biologique de la France et de la Belgique, Vol. 37, 1952, pp. 1-158.

[8] T. N. Loi, "Peuplements Animaux et Vegetaux du Substrat des Intertidal de la Baie de Nha Trang (Vietnam)," Memories Institute of Oceanography, Vol. 11, 1967, pp. 1-236.

[9] C. Cheung, "Vietnam Marine Conservation," Coastal Management in Tropical Asia, Vol. 4, 1994, pp. 21-23.

[10] Y. Y. Latypov, "Scleractinian Corals of Vietnam. Thamnasteriidae, Astroceniidae, Pocilloporidae, Dendrophylliidae)," Nauka, Moscow, 1990.

[11] Y. Y. Latypov, "Coral Reefs of Vietnam," Nauka, Moscow, 2007.

[12] Y. Y. Latypov, "Species Composition and Structure of Coral Community of a Platform Reef at Bach Long Vi Island in the South China Sea," Russian Journal of Marine Biology, Vol. 34, No. 4, 2008, pp. 249-253. doi:10.1134/S1063074008040068 
[13] L. V. Ken, "The Stony Corals in the Sea of Vietnam, Marine Environment and Resources, (1986-1990)," Scientific and Technic Publishing House, Hanoi, 1991, pp. 127-135.

[14] N. H. Yet, "Characteristic Species and Coral Reef Structures Fishermen Dao (Spratly Islands)," Resources and Marine Environment, Vol. 4, 1997, pp. 299-313.

[15] J. A. Hubbard, "Scleractinian Corals Behavior in Calibrated Carrent Experiment: An Index to Their Distribution Patterns," Proceeding of 2nd International Coral Reef Symposium, Vol. 2, 1974, pp. 107-126.

[16] M. Pichon, "Dynamic of Benthic Communities in the Coral Reefs of Tule'ar (Madagaskar): Succession and Transformation of the Biotops Trough Reef Tract Evolution," Proceeding of 2nd International Coral Reef Symposium, Vol. 2, 1974, pp. 55-68.

[17] Y. Loya, "The Red Sea Coral Stylophora Pistillata is an Rstrategist," Nature, Vol. 259, 1976, pp. 478-480. doi:10.1038/259478a0

[18] Y. Y. Latypov, "Community Structure of Scleractinian Reefs in the Baitylong Archipelago (South China Sea)," Asian Marine Biology, Vol. 12, 1995, pp. 27-37.

[19] Y. Y. Latypov, "Coral Reefs of the Gulf of Tonkin," Vestnik DVO RAN, No. 2, 1997, pp. 92-98.

[20] S. A. Wainwright, "Reef Communities Visited by the Israel South Red Expedition, 1962," Bulletin of Sea Fishery Research. Station, Vol. 8, 1965, pp. 40-53.

[21] Y. Y. Latypov, "Species Composition and Distribution of Scleractinians in Reefs of the Khanh Hoa Province (South Vietnam)," Biologia Morya, No. 6, 1982, pp. 5-12.

[22] B. V. Preobrazhenskii, "Sovremennye Rify," Nauka, Moscow, 1986.

[23] Y. Y. Latypov, "Coral Communities of the Namsu Islands (Gulf of Siam, South China Sea)," Marine Ecology Progress Series, Vol. 29, 1986, pp. 261-270. doi:10.3354/meps029261

[24] Y. Y. Latypov, "Scleractinian Corals of South Vietnam," The Soviet Journal of Marine Biology, Vol. 13, No. 5, 1987, pp. 246-252.

[25] N. H. Yet and L. V. Ken, "Some Data on Species Composition and Distribution of Scleractinian Corals in Ha Long Bay," Journal of Biology, Vol. 18, 1996, pp. 7-13.

[26] Y. I. Sorokin, "Ecosystems of Coral Reefs," Nauka. Moscow, 1990

[27] Y. Y. Latypov, "Reef-Building Corals of Vietnam as a Part of the Indo-Pacific Reef Ecosystem," Russian Journal of Marine Biology, Vol. 31, Supplement 1, 2005, pp. S34-S40. doi:10.1007/s11179-006-0013-5

[28] Y. Y. Latypov and N. I. Selin, "Coral Communities of Barrier Reefs of Vietnam," Russian Journal of Marine Biology, Vol. 34, No. 3, 2008, pp. 143-150. doi:10.1134/S1063074008030012

[29] Y. Y. Latypov, "Reef-Building Corals and Reefs of Vietnam: 1. The Gulf of Thailand," Russian Journal of Marine Biology, Vol. 29, Supplement 1, 2003, pp.

\section{S22-S33. doi:10.1023/B:RUMB.0000011714.71499.75}

[30] Y. Y. Latypov, "Reef-Building Corals and Reefs of Vietnam: 2. The Gulf of Tonkin," Russian Journal of Marine Biology, Vol. 29, Supplement 1, 2003, pp. S34-S45. doi:10.1023/B:RUMB.0000011715.09137.b0

[31] Y. Y. Latypov and P. Q. Long, "The Common Hard Corals of Vietnam," Ministry of Agriculture and Rural Development, Hanoi, 2010.

[32] S. T. Vo, N. H. Yet and P. M. Alino, "Coral and Coral Reefs in the North of Spratly Archipelago the Results of RP-VN JOMSRE-SCS 1996," Proceeding of Scientific Conference on Philippines-Vietnam JOMSRE 1996, Hamoi, 22-23 April 1997, pp. 87-101.

[33] World Wildlife Fund, "Vietnam Marine Conservation Southern Survey Team," Survey Report on the Biodiversity Resourse Utilization and Conservation Potential of Coto Islands, Switzerland, 1994.

[34] M. B. Best, B. W. Hoeksema, W. Moka, et al., "Recent Scleractinian Corals Species Collected during the Snellius-II Expedition in Eastern Indonesia," Netherland Journal of Sea Research, Vol. 23, No. 2, 1989, pp. 107-115.

[35] J. E. N. Veron and G. Hodgson, "Annotated Checklist of the Hermatypic Corals of the Philippines," Pacific Science, Vol. 43, No. 3, 1989, pp. 234-287.

[36] J. E. N. Veron, "Corals in Space and Time: The Biogeography and Evolution of the Scleractinia," Australian Institute of Marine Sciences, Townsville, 1995, p. 321.

[37] J. E. N. Veron and L. M. Marsh, "Records and Annotated Check List of the Hermatypic Corals of Western Australia," Records of the Western Australia Museum, 1988, p. 29.

[38] Y. Y. Latypov, "Benthic Communities of Coral Reefs of Tho Chu Island (Gulf of Siam, South China Sea)," Russian Journal of Marine Biology, Vol. 25, No. 3, 1999, pp. 233-241.

[39] Y. Y. Latypov, "Macrobenthos Communities on Reefs of the an Thoi Archipelago of the South China Sea," Russian Journal of Marine Biology, Vol. 26, No. 1, 2000, pp. 18-26. doi:10.1007/BF02759489

[40] K. Sakai, T. Yeemin, A. Svidvong, et al., "Distribution and Community Structure of Germatypic Corals in the Sichang Islands, Inner Part of the Gulf of Thailand," Galaxea, Vol. 5, 1986, pp. 27-74.

[41] H. Mergner, "Quantitative Okologische Analyse Eines Rifflagunenareals bei Agaba (Golf von Agaba, Rotes Meer)," Helgoland Wissenschsft Meeresuntersuch, Vol. 32, No. 4, 1979, pp. 476-507. doi:10.1007/BF02277991

[42] S. Dollar, "Wave Stress and Coral Community Structure in Hawaii," Coral Reefs, Vol. 1, No. 2, 1982, pp. 71-81. doi:10.1007/BF00301688

[43] C. F. Dai, "Patterns of Coral Distribution and Benthic Space Partitioning on the Fringing Reefs of Southern Taiwan," Marine Ecology, Vol. 14, No. 3, 1993, pp. 185-204. doi:10.1111/j.1439-0485.1993.tb00479.x

[44] W. D. Liddel and S. L. Ohlhorst, "Patterns of Reef Coral Community Structure. North Jamaica," Bulletin of Biol- 
ogy, Vol. 40, 1987, pp. 311-329.

[45] Y. Y. Latypov, "Reefs and Communities of Sclerac- tinians of the Island (South Vietnam)," In: Biology of Coastal Waters of Vietnam: Hydrobiological Investigations of Intertidal and Subtidal Zones of South Vietnam, DVO AN SSSR, Vladivostok, 1988, pp. 11-19.

[46] Y. Loya, "Effects of Water Turbidity and Sedimentation on the Community Structure of Puerto Rican Corals," Bulletin Marine Sciences, Vol. 26, No. 4, 1976, pp. 450-466.

[47] J. W. Porter, "Autotrophy, Heterotrophy and Recourses Partitioning in Caribbean Reef-Building Corals," American Naturalist, Vol. 110, No. 975, 1976, pp. 731-742. doi: $10.1086 / 283100$

[48] T. Tomascik and F. Sander, "Effect of Eutrophication on Reef-Building Corals. 3. Reproduction of the ReefBuilding Coral Porites Porites," Marine Biology, Vol. 94, No. 1, 1987, pp. 77-94.
doi:10.1007/BF00392901

[49] B. V. Preobrazhenskii, "Morphology and Paleoecology of Tabulate Corals," Nauka, Moscow, 1982

[50] Y. Y. Latypov, "Benthic Communities of the Corals Reef of the Kondao Islands in the South China Sea," Russian Journal of Marine Biology, Vol. 20, No. 3, 1994, pp. 136-143.

[51] S. Ekman, "Zoogeography of the Sea," Sidgwik and Jackson, London, 1953.

[52] E. G. Stehli and I. W. Wells, "Diversity and Age Patterns in Hermatypic Corals," Systematic Zoology, Vol. 20, No. 20, 1971, pp. 115-126. doi:10.2307/2412052

[53] A. N. Malyutin and Yu. Ya. Latypov, "Distribution of Corals and Biogeographic Zonation of the Shelf of Vietnam,” Biologia Morya, No. 4, 1991, pp. 26-35. 